\title{
MASS LOSS IN PRE-MAIN-SEQUENCE STARS VIA CORONAL MASS EJECTIONS AND IMPLICATIONS FOR ANGULAR MOMENTUM LOSS
}

\author{
Alicia N. Aarnio ${ }^{1}$, Sean P. Matt ${ }^{2}$, and Keivan G. Stassun ${ }^{3,4}$ \\ ${ }^{1}$ Astronomy Department, University of Michigan, 830 Dennison Building, 500 Church Street, Ann Arbor, MI 48109, USA; aarnio@ umich.edu \\ ${ }^{2}$ Laboratoire AIM Paris-Saclay, CEA/Irfu Université Paris-Diderot CNRS/INSU, F-91191 Gif-sur-Yvette, France \\ ${ }^{3}$ Department of Physics and Astronomy, Vanderbilt University, Nashville, TN 37235, USA \\ ${ }^{4}$ Department of Physics, Fisk University, Nashville, TN 37208, USA \\ Received 2012 June 21; accepted 2012 September 27; published 2012 October 30
}

\begin{abstract}
We develop an empirical model to estimate mass-loss rates via coronal mass ejections (CMEs) for solar-type pre-main-sequence (PMS) stars. Our method estimates the CME mass-loss rate from the observed energies of PMS X-ray flares, using our empirically determined relationship between solar X-ray flare energy and CME mass: $\log \left(M_{\mathrm{CME}}[\mathrm{g}]\right)=0.63 \times \log \left(E_{\mathrm{flare}}[\mathrm{erg}]\right)-2.57$. Using masses determined for the largest flaring magnetic structures observed on PMS stars, we suggest that this solar-calibrated relationship may hold over 10 orders of magnitude in flare energy and 7 orders of magnitude in CME mass. The total CME mass-loss rate we calculate for typical solar-type PMS stars is in the range $10^{-12}-10^{-9} M_{\odot} \mathrm{yr}^{-1}$. We then use these CME mass-loss rate estimates to infer the attendant angular momentum loss leading up to the main sequence. Assuming that the CME outflow rate for a typical $\sim 1 M_{\odot}$ T Tauri star is $<10^{-10} M_{\odot} \mathrm{yr}^{-1}$, the resulting spin-down torque is too small during the first $\sim 1$ Myr to counteract the stellar spin-up due to contraction and accretion. However, if the CME mass-loss rate is $\gtrsim 10^{-10} M_{\odot} \mathrm{yr}^{-1}$, as permitted by our calculations, then the CME spin-down torque may influence the stellar spin evolution after an age of a few Myr.
\end{abstract}

Key words: stars: activity - stars: evolution - stars: mass-loss - stars: pre-main sequence - stars: solar-type

Online-only material: color figures

\section{INTRODUCTION}

Solar-type pre-main-sequence (PMS) stars typically evince magnetic activity in a variety of forms, including strong and time-variable coronal emission at X-ray wavelengths (e.g., Güdel 2008; Preibisch et al. 2005), strong and time-variable chromospheric emission at ultraviolet wavelengths (e.g., Yang et al. 2012; Petrov et al. 2011; Valenti et al. 1993) and in chromospheric emission lines (such as $\mathrm{H} \alpha$; e.g., White \& Basri 2003; Fernandez et al. 1995; Walter \& Kuhi 1981), large spotmodulated photometric variability (e.g., Herbst et al. 2007; Stassun et al. 1999; Herbst et al. 1994), and non-thermal polarized radio continuum emission is a classic indicator of magnetic activity (e.g., the PMS star Hubble 4; Skinner 1993). These observations have motivated direct Zeeman measurements revealing field strengths of typically a few kG (e.g., Johns-Krull 2007; Reiners \& Basri 2007; Johns-Krull et al. 2004). The strong magnetic fields associated with this ubiquitous activity are thought to be central to a number of key physical processes in PMS stars, including specifically the transfer of mass and of angular momentum from and to the circumstellar environment.

X-ray activity observed in PMS stars bears a striking resemblance to solar X-ray activity, albeit scaled up by several orders of magnitude in both energy and frequency of occurrence (e.g., Peres et al. 2004, 2006; Getman et al. 2005; Audard et al. 2007). Generally, PMS X-ray flares are well described by standard solar flare models (e.g., Reale et al. 1997), though in the case of some exceptionally long-lived flares it has been necessary to add self-eclipse of loop emission by the star (i.e., Skinner et al. 1997; Johnstone et al. 2012). In addition, with the exception of a few very strongly accreting systems where the X-ray variability is at least in part accretion driven (e.g., V1647 Ori; Kastner et al. 2004; Hamaguchi et al. 2012), large-scale studies of simultaneous X-ray and optical variability in PMS stars show that the X-ray variability is principally caused by coronal activity (Stassun et al. 2006, 2007). Young, solar-type stars appear, then, to have flaring magnetic field configurations that behave like, and can be interpreted as, scaled-up solar type flaring fields (see also Feigelson et al. 2007, for a recent overview).

During the early, active accretion phase of a PMS star's evolution (first few Myr; e.g., Haisch et al. 2001), the strong stellar field may be important in governing the interaction of the star with its protoplanetary disk. This magnetic star-disk interaction likely controls the funneling of circumstellar material from the disk onto the star (e.g., Shu et al. 1994), is likely central to the launching of outflows including jets and winds (e.g., Hayashi et al. 1996; Matt \& Pudritz 2005a), and therefore is likely a key mediator in the net flux of mass and angular momentum onto and away from the young star (e.g., Matt \& Pudritz 2005a; Matt et al. 2010; Orlando et al. 2011).

Even after the phase of active accretion subsides, scaled-up solar-type winds and elevated solar-type X-ray activity do not decline to present-day solar levels until approximately $1 \mathrm{Gyr}$ (Wood 2004; Wood et al. 2005; Guedel et al. 1997), potentially affecting the ongoing evolution of the star's angular momentum and the circumstellar environments of young planets (e.g., Vidotto et al. 2010).

Extremely large magnetic loops-extending out to tens of stellar radii from the stellar surface-have been inferred among the most powerful X-ray flaring PMS stars from the Chandra Orion Ultra-deep Project (COUP) sample (e.g., Favata et al. 2005; Massi et al. 2008; Skelly et al. 2008), implying very large magnetic lever arms that are potentially able to shed significant angular momentum (Aarnio et al. 2009, 2012). While it was at first appealing to picture these large magnetic loops as being linked to circumstellar disks, perhaps either arising from a star-disk reconnection event (Orlando et al. 2011) or simply maintaining loop stability via anchoring to the disk, Aarnio et al. 
(2010) showed that the majority of these stars lack massive disks within reach of the loops. Indeed, earlier work by Getman et al. (2008) came to similar conclusions, inferring from the apparent absence of large magnetic loops among stars with close-in disks that perhaps it is the absence of the truncation by an inner dust disk which allows these loops to be so large in the first place.

On the Sun, the eruption of such magnetic structures can drive coronal mass ejections (CMEs), which are observed in Thomson-scattered optical light (cf. Vourlidas \& Howard 2006). While the faint scattered light of CMEs cannot yet be directly detected from most other stars, there have been recent observations of young stars with the Far-Ultraviolet Spectroscopic Explorer satellite (Leitzinger et al. 2011) and the detection of a large flare-associated mass ejection in the PMS star Z CMa (Stelzer et al. 2009; Whelan et al. 2010). More generally, CMEs are expected from low-mass stars by analogy to the Sun as well as from basic escape velocity considerations for the flaring material; indeed, CMEs are believed to have been detected on $\mathrm{M}$ and $\mathrm{K}$ dwarfs from Balmer line asymmetries, transient spectral line absorption components, and EUV dimmings (cf. Leitzinger et al. 2011 and references therein). In particular, the extreme X-ray flares observed on PMS stars are expected to have associated extreme CMEs (e.g., Aarnio et al. 2011), the ramifications of which-for the evolution of the star, for the protoplanetary environment, and for the star's angular momentum evolution-depend on the resulting mass-loss rate and the evolution of that mass loss over the course of the star's PMS evolution.

A number of studies have used emission line tracers to empirically estimate the mass-loss rates from PMS winds during the actively accreting, classical T Tauri star (CTTS) phase (see, e.g., Kwan et al. 2007; Edwards et al. 2006, 2003 and references therein), and such accretion-powered PMS winds have been explored in detail theoretically (e.g., Matt \& Pudritz 2005a, 2008a; Cranmer 2008, 2009; Matt \& Pudritz 2008b; Matt et al. 2010). Meanwhile, reliable empirical measures of mass-loss rates for non-accreting PMS stars, particularly from impulsive CME-type events, are virtually nonexistent, and simulations of winds from non-accreting, weak-lined T Tauri stars (WTTSs) have only recently been investigated (Vidotto et al. 2009a, 2009b, 2010).

Here, we aim to make first empirical estimates of massloss rates for PMS stars resulting from CMEs, and then use these mass-loss rate estimates to infer the attendant angular momentum loss leading up to the main sequence. Specifically, we develop a method to estimate CME mass-loss rates from observed PMS X-ray flares, calibrated to solar observations and models, and then calculate the resulting braking torques in the context of PMS stellar evolutionary models.

We begin by summarizing our previous work to establish an empirical relationship between X-ray flare flux and CME mass for the Sun, and describe how we then extend that relationship to solar-type PMS stars (Section 2). In Section 3, we use our empirical flare/CME relationship to derive CME mass-loss rates for PMS stars both empirically (using directly observed flare properties) and analytically (using functional representations of flare properties), and furthermore test our methodology on the Sun, verifying that we are able to recover its well-measured CME mass-loss rate from its observed X-ray flares. Next, in Section 4, we calculate the attendant torque on PMS stars exerted by the CMEs, and assess the likelihood that these braking torques may serve to prevent the stars from spinning up as they contract toward the main sequence. Finally, we discuss

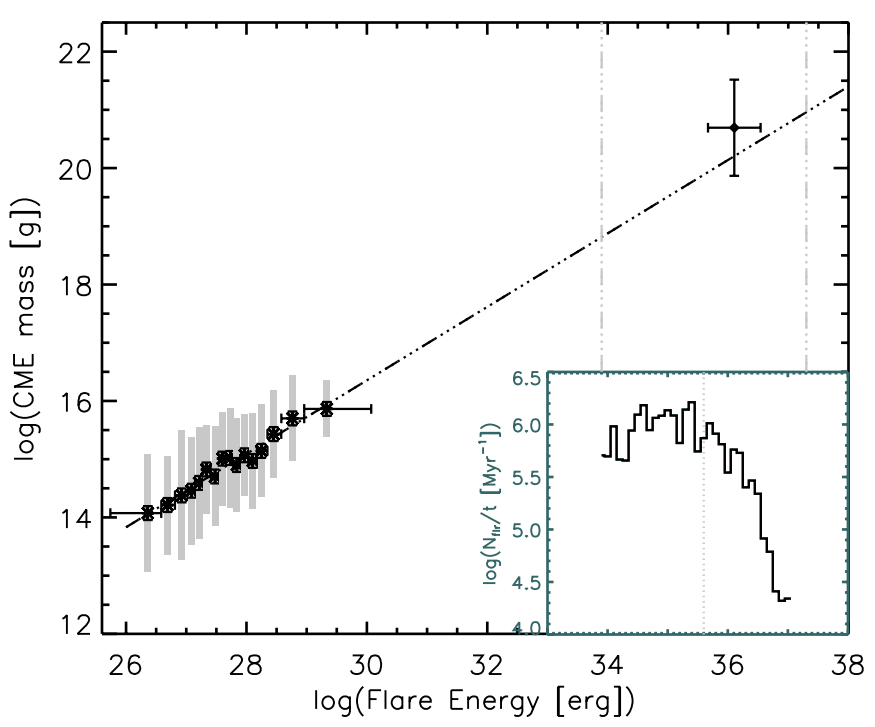

Figure 1. Solar relationship between flare energy and CME mass, transformed from the flux/mass relationship of Aarnio et al. (2011). The line is a fit to the solar data over the energy range of observed solar flares, $10^{26}-10^{30} \mathrm{erg}$, and then extrapolated to the regime of $\mathrm{T}$ Tauri star flare energies (represented by vertical lines). The single point at upper right shows the mean CME mass and flare energy for the $32 \mathrm{~T}$ Tauri star mega-flares observed by COUP (Favata et al. 2005), and the error bars represent the standard deviations of those mean values. The extrapolated solar relationship fits this point well. Inset: the energy distribution of flare rates for T Tauri stars observed by COUP (Albacete Colombo et al. 2007). The vertical dotted line at $10^{35.6}$ erg represents the energy above which the observed flare sample is complete.

our findings in Section 5 and summarize our conclusions in Section 6.

\section{A SOLAR-CALIBRATED RELATIONSHIP BETWEEN PMS X-RAY FLARES AND CME MASSES}

In Aarnio et al. (2011), we cross-matched 10 years of solar flare and CME observations in order to determine, for associated flares and CMEs, whether there are correlations between flare and CME properties. Approximately 1000 solar flares and CMEs were found to be spatially and temporally associated. The resulting relationship between flare flux and CME mass is well fit by a single power law of the form:

$$
\log (M[\mathrm{~g}])=18.7+0.7 \times \log \left(F\left[\mathrm{~W} \mathrm{~m}^{-2}\right]\right),
$$

where $M$ is the CME mass and $F$ the flare flux. Flare flux and CME mass hold this log-linear relationship over a few dex in flux and mass.

Solar flares are, by convention, classified by their peak X-ray flux, while for stellar X-ray flare observations, flare luminosities are reported. Therefore, to relate our solar flare flux/CME mass relationship to observations of PMS stellar flares, we convert the solar flare X-ray fluxes into energies, and reframe the CME mass/flare flux relationship into a CME mass/flare energy relationship. To determine solar flare energy, we integrate the flux of the solar X-ray flare light curves from flare start to end time. For simplicity, we approximate the flares by their peak energy and assume a linear decay. Specifically, each flare's total energy output equals one half of the observed peak flux, times the observed flare duration, times $4 \pi(1 \mathrm{AU})^{2}$. Our final solar CME mass/flare energy relationship is shown in Figure 1, and it has the functional form:

$$
M=K_{\mathrm{M}} E^{\beta}
$$


in this and all following cases, $M$ will denote the CME mass and $E$ the flare energy. $K_{\mathrm{M}}$ is $(2.7 \pm 1.2) \times 10^{-3}$ in cgs units, and $\beta=0.63 \pm 0.04$.

In adopting this relationship, we take advantage of the empirical correlation between associated flares and CMEs, but we make no assumption or statement of a causal relationship between flares and CMEs. Indeed, it is well established that not every flare is associated with a CME, and not every CME is associated with a flare. Aarnio et al. (2011) found that $13 \%$ of CMEs are flare associated and $\sim 9 \%$ of flares are CME associated; this lack of correspondence is at least partially driven by observational biases, and any correction for over- or underestimating the number of CMEs associated with flares is likely less than approximately a factor of two. The most energetic solar flares, however, are the most likely to be CME-associated, and these CMEs are the most massive (Andrews 2003; Yashiro et al. 2005, 2006; Aarnio et al. 2011, to name but a few studies on the matter). Since young stars' flares are very powerful with respect to solar flares and the most energetic solar flares have the highest rate of CME coincidence, we could suppose that a flare/CME association probability is close to unity for TTS flares.

Since we cannot yet directly observe CMEs on young stars, we will use our solar-calibrated relationship (Equation (2)) as applied to X-ray flare observations of young stars to determine limits on the mass losses from young stars via solar-analog CMEs. However, PMS X-ray flare energies are five to seven orders of magnitude stronger than solar flares, requiring a large extrapolation of the solar flare/CME relationship. Therefore, it is prudent to demonstrate that the likely masses of CMEs associated with the extreme X-ray flare energies observed on PMS stars are in fact consistent with the solar flare/CME relationship. To do this, we have estimated the masses associated with the 32 most powerful PMS X-ray flares observed in Orion by COUP (Favata et al. 2005). Strictly speaking, these masses represent the masses of post-flaring loops, which might not be equivalent to the CME masses, but in any case by analogy to the Sun should be related to the CME masses.

We use the flaring loop physical parameters for these 32 extreme PMS flares as derived by Favata et al. (2005), who used the solar-calibrated uniform cooling loop (UCL) model of Reale et al. (1997) to infer the flaring loop lengths and their densities, the latter inferred from the flare peak emission measures. We adopt the typically assumed cylindrical loop shape and the typically assumed loop aspect ratio (radius/length $=0.1$; Reale et al. 1997), permitting the flaring loop volumes to be calculated. The loop masses then follow from the volumes and densities. In this way, we obtain flaring loop masses ranging from $10^{19} \mathrm{~g}$ to $10^{22} \mathrm{~g}$ for the Favata et al. (2005) sample. These 32 flaring loop masses and their associated flare energies are shown in Figure 1 as the point with error bars at the far upper right, representing the mean and standard deviation of the sample. The large scatter of $\sim 1$ dex in the masses is likely due in part to the simplistic assumptions of the UCL model upon which the mass estimates are based. For example, the UCL model assumes that a single magnetic loop is involved in the observed X-ray flare, while on the Sun, arcades of smaller loops are often involved in a given flaring "event." Väänänen et al. (2009) suggest that the height of such loop arcades can differ from the UCL inferred loop heights by factors of 2-10, consistent with the scatter in loop masses we have estimated in Figure 1. In any case, the extreme PMS CME masses so inferred are consistent to within a factor of 10 with that predicted by the solar-calibrated flare/CME relationship.
Note that we have not adjusted the fit from the solar data, but merely extrapolated it, which lends strong support to the use of this same solar-calibrated flare/CME relationship over the full range of solar to TTS flare energies.

\section{MASS LOSS VIA CMEs}

Having established the form of the relationship between flare energy and CME mass, in this section we derive total CME massloss rates for PMS stars, both (1) empirically using the observed distribution of flare energies directly (Sections 3.1.1 and 3.2.1) and (2) analytically using the observed flares to describe a functional form for the distribution of flare energies (Sections 3.1.2 and 3.2.2). First, however, we apply our methodology to the solar case as a confirmation that our method recovers the observed CME mass-loss rate for the Sun.

\subsection{Solar Case}

We start by demonstrating our method for the solar case as a benchmark. For the sun, we have a measured CME mass distribution, from which we can directly calculate a CME massloss rate to compare to the mass-loss rates that we infer from the X-ray flares.

In the Large Angle and Spectrometric Coronagraph (LASCO) CME database (Gopalswamy et al. 2009), 13,862 CMEs are reported from 1996 to 2006, spanning almost a full solar activity period including minimum and maximum. Of those, $6733 \mathrm{CMEs}$ have well-constrained mass measurements, and an additional 1395 have masses flagged as highly uncertain. We show the full rate distribution of reported CME masses (number of CMEs per year as a function of CME mass), including both wellconstrained and highly uncertain CME masses, in Figure 2 (upper middle panel). We have included here the additional halo CMEs (those projected toward the Earth, or which are so wide as to appear as if they were) for which Aarnio et al. (2011) estimated masses.

Summing up this entire distribution gives a CME mass-loss rate of $7.8 \times 10^{-16} M_{\odot} \mathrm{yr}^{-1}$. Put another way, between 1996 and 2006, the Sun shed at least $1.563 \times 10^{18} \mathrm{~g} \mathrm{yr}^{-1}$ via CMEs. This value represents a lower limit to the mass-loss rate via CMEs from the Sun, as just under half of the reported CMEs have measured masses and there may be CMEs that escape observation, especially at lower masses. Aarnio et al. (2011) found 1153 of the CMEs with measured masses to be associated with flares (the masses of these CMEs are also shown in Figure 2, upper middle panel); these CMEs were used to derive the relationship between flare energy and CME mass (Equation (2)). Even though only $\sim 15 \%$ of the CMEs with measured masses are associated with flares, these flare-associated CMEs constitute $40 \%$ of the total observed CME mass-loss rate.

The above directly measured CME mass-loss rate thus provides a benchmark that our flare-inferred CME mass-loss rate below should reproduce. To compare the CME mass-loss rate that we will infer from flares below to the observed solar case, we will find it convenient to represent the solar CME mass distribution (Figure 2, upper middle panel) as a differential CME mass distribution $(d N / d M)$, and this is shown in Figure 2, in the lower middle panel.

\subsubsection{Empirically Determined Solar CME Mass-loss Rate}

To estimate the solar CME mass-loss rate from flares, we require a flare energy distribution to use with our empirical relation between flare energy and CME mass (Equation (2)). 

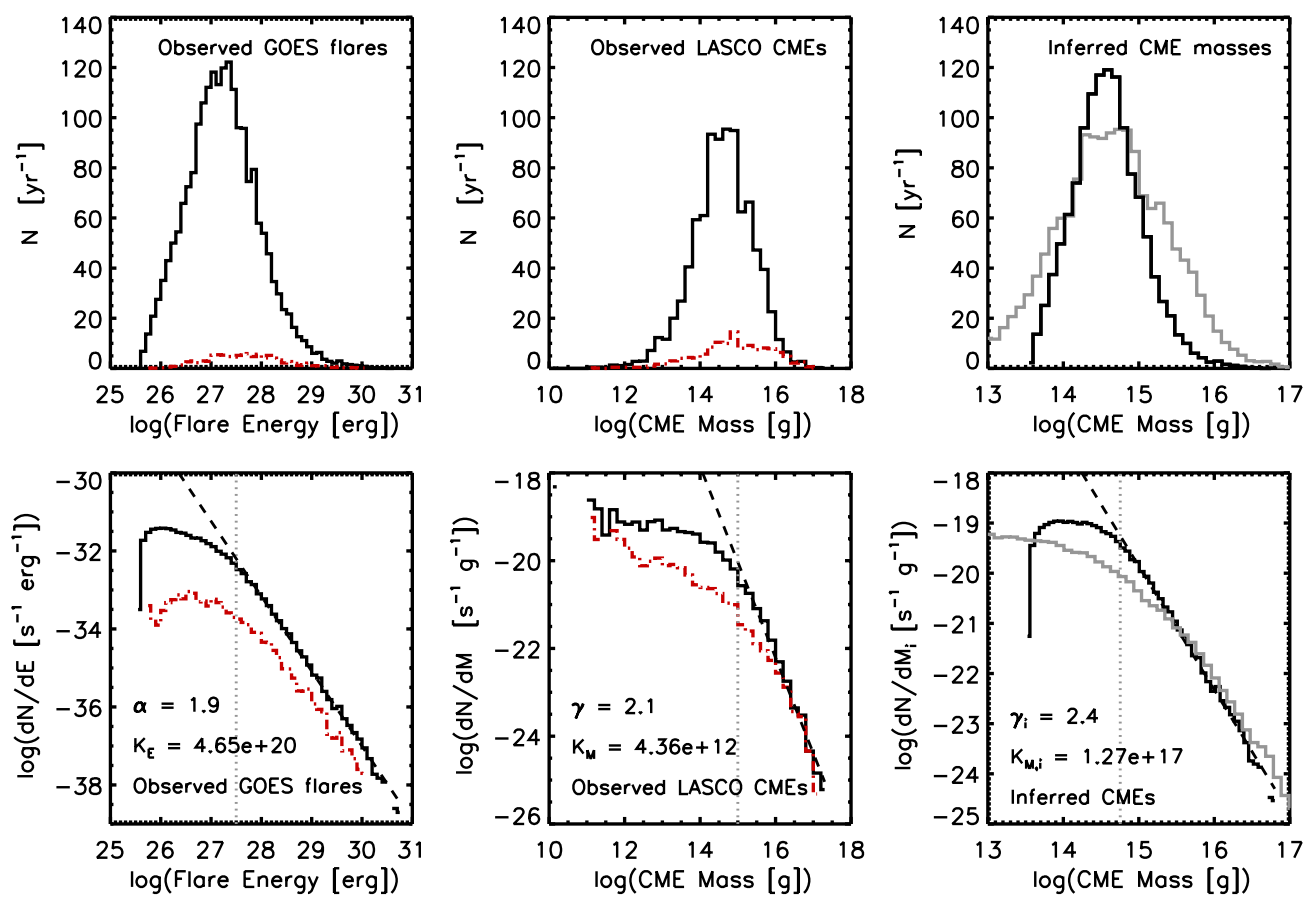

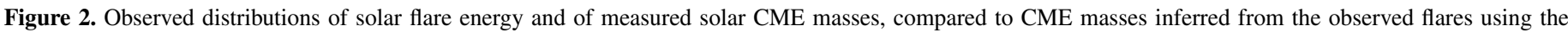

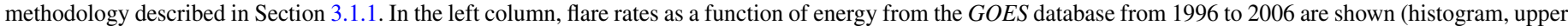

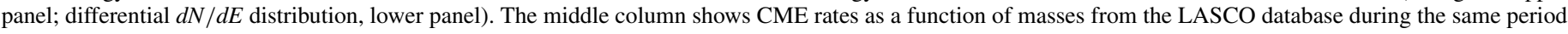

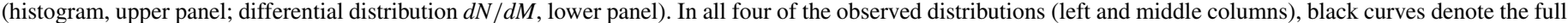

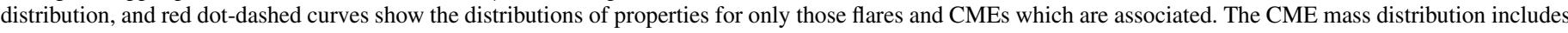

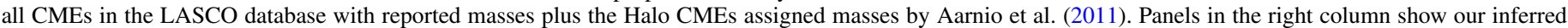

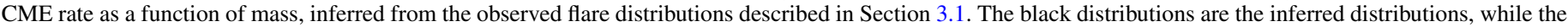

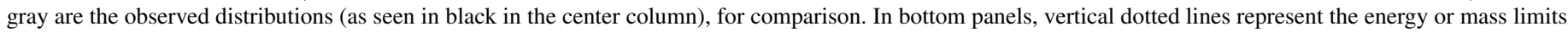
above which the distributions are approximately complete.

(A color version of this figure is available in the online journal.)

In order to do this as we will below for TTSs, we can determine a solar flare frequency $f_{\text {flr }}$ as done by Albacete Colombo et al. (2007) for the COUP data. The GOES satellite recorded 22,674 solar flares from 1996 to 2006, so the total $f_{\text {flr }}=0.065 \mathrm{ks}^{-1}$ (this value is 0.0007 for the flares observed by the COUP; see Section 3.2.1 below). The energy distribution of observed flare rates (number per year as a function of energy) is shown in Figure 2, in the top left panel. As with the CMEs, we convert this flare energy distribution into a differential energy distribution $(d N / d E$; Figure 2, lower left panel) and fit a power law of the form:

$$
\frac{d N}{d E}=K_{\mathrm{E}} E^{-\alpha}
$$

our fit above an estimated completeness threshold (see below) of $E_{\text {cut }}=10^{27.5} \mathrm{erg}$ (vertical line in Figure 2, lower left panel) gives $\alpha=1.92( \pm 0.02)$ and $K_{\mathrm{E}}=4.7 \times 10^{20}( \pm 3 \%)$ in cgs units. Our value for $\alpha$ is consistent with those reported in the literature: Hudson (1991) reports $\alpha=1.8$ in $d n / d W=A W^{-\alpha}$, where $W$ is the total energy radiated by the flare, and below the GOES detection limit, RHESSI data indicate that this distribution extends to lower flare energies and continues to exhibit powerlaw behavior, with a slope of $\sim 1.5$ (Christe et al. 2008).

Next, we compute the inferred mass distribution of $\mathrm{CME}$ rates by using Equation (2) to convert the energy bins in the flare energy distribution (Figure 2, left bottom panel) into mass bins, i.e., $d N / d E \rightarrow d N / d M$, where

$$
\frac{d N}{d M}=\frac{d N}{d E} \times \frac{d E}{d M}
$$

and where $d E / d M$ is given by Equation (2). The resulting differential CME mass distribution is shown in Figure 2, right bottom panel, and the corresponding non-differential distribution is shown in the right upper panel for ease of comparison with the other distributions shown in the top panels. The total mass in this empirically determined mass distribution is $\dot{M}_{\text {cme }}=\sum d N / d M M=1.558 \times 10^{18} \mathrm{~g} \mathrm{yr}^{-1}$, which impressively is equal to the total observed LASCO solar CME mass-loss rate (Section 3.1) to within less than a percent.

This is reassuring that the CME mass-loss rate inferred directly from observed flares is able to reproduce the directly measured CME mass-loss rate. At the same time, as the right column of Figure 2 shows, our inferred mass distribution reflects a couple of known biases: first, we underpredict the highest mass CMEs because our flare-CME relationship does not take into account the association probabilities of flares and CMEs that increase with greater flare energy. Second, we overpredict the observed occurrence of lower mass CMEs, likely because of a bias against the detection of the lowest energy flares (in Figure 2, $d N / d E$ "turns over" at lower energies).

Thus, while our empirical flare-inferred CME mass-loss rate appears to work well at reproducing the directly measured CME mass-loss rate, we can attempt to account for those flares and CMEs that are missed observationally by describing the observed CME rate analytically, as we now discuss.

\subsubsection{Analytically Determined Solar CME Mass-loss Rate}

In an effort to account for the observational biases mentioned above-most importantly, the missed low-energy flares 

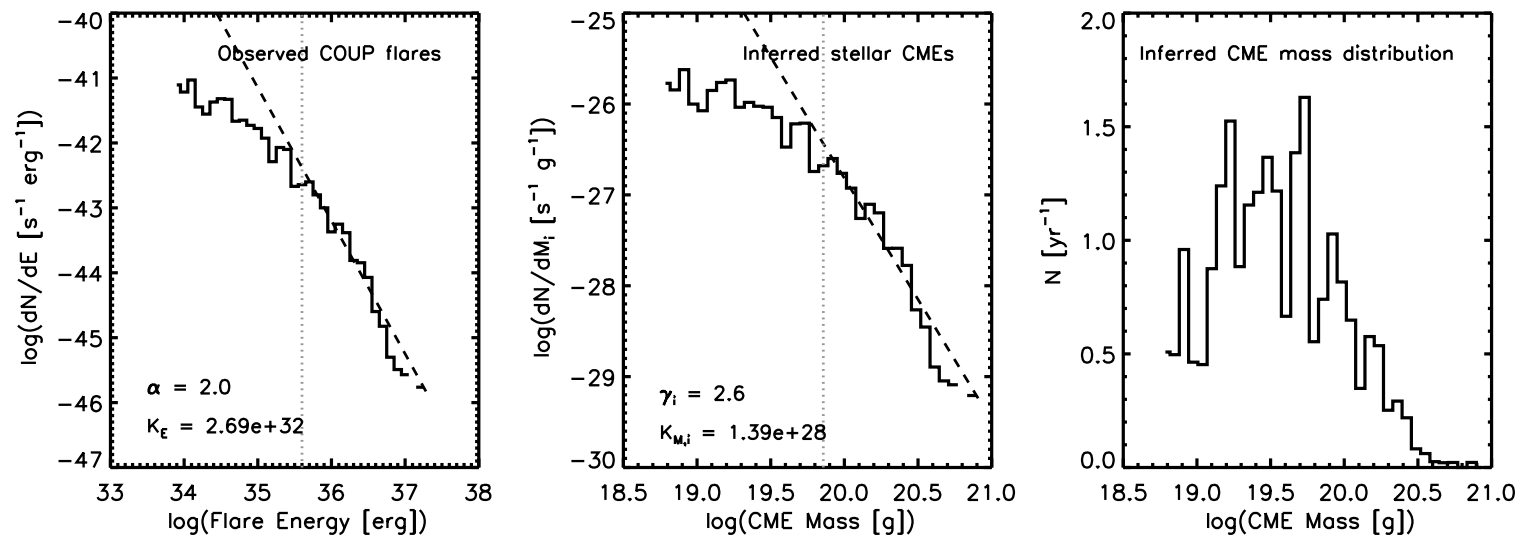

Figure 3. Flare and CME distributions for PMS stellar sample from the COUP. The left panel is the flare frequency distribution of Albacete Colombo et al. (2007), expressed in terms of number of flares of a given energy per time per energy bin $(d N / d E)$. In the middle panel, we show our inferred TTS CME mass distribution: we have taken the product of $d N / d E$ from the left panel and $d E / d M$ derived here in Section 2 to arrive at $d N / d M$. Then, in the right panel, we show the stellar CME mass distribution were it observed over the same time span as the LASCO CMEs (compare to Figure 2, upper middle panel).

and therefore the lowest mass CMEs-we can replace the directly observed flare distribution with analytical fits to the observationally complete parts of our flare energy and CME mass distributions, and assuming a simple power law of the form:

$$
\begin{aligned}
\dot{M}_{\mathrm{CME}} & =\int_{M_{\min }}^{M_{\max }} \frac{d N}{d M_{\mathrm{i}}} M_{\mathrm{i}} d M \\
& =\frac{K_{\mathrm{M}, \mathrm{i}}}{2-\left(\gamma_{\mathrm{i}}\right)}\left(M_{\max }^{2-\gamma_{\mathrm{i}}}-M_{\min }^{2-\gamma_{\mathrm{i}}}\right),
\end{aligned}
$$

where $M_{\mathrm{i}}$ represents our flare-inferred CME masses from Equation (2), and

$$
\begin{aligned}
\frac{d N}{d M_{\mathrm{i}}} & =K_{\mathrm{M}, \mathrm{i}} M_{\mathrm{i}}^{-\gamma_{\mathrm{i}}} \\
& =\frac{K_{\mathrm{E}}}{\beta K_{\mathrm{M}, \mathrm{i}}^{(\alpha+\beta-1) / \beta+1}} M_{\mathrm{i}}^{(\alpha+\beta-1) / \beta} .
\end{aligned}
$$

Equation (5) is the integral of the inferred CME mass distribution, i.e., the integrated power law of Figure 2, bottom right panel, and $K_{E}$ and $\beta$ are from Equation (2) (the fitted values of $K_{\mathrm{M}, \mathrm{i}}$ and $\gamma_{\mathrm{i}}$ are reported in the plot legend). For the upper limit of the integral, we take the highest solar CME mass observed, $M_{\max } \sim 6 \times 10^{16} \mathrm{~g}$. To determine the value of $M_{\min }$, we can either use the observational completeness limit of $d N / d E$ or else simply the minimum observed CME mass. We begin with the clearest observational constraint, the completeness limit of $d N / d E$. Plugging $E_{\text {cut }}\left(10^{27.5} \mathrm{erg}\right)$ into the flare energy/CME mass relationship (Equation (2)), we find a corresponding $M_{\text {min }}$ of $\sim 10^{15} \mathrm{~g}$. This represents a very conservative minimum mass; clearly, the observed CME mass distribution contains a significant number of CMEs less massive than $\sim 10^{15} \mathrm{~g}$. The resulting mass-loss rate, then, as calculated with Equation (5), is $6.6 \times$ $10^{-16} M_{\odot} \mathrm{yr}^{-1}$. Were we to simply set $M_{\min }$ equal to the minimum mass CME inferred, $\sim 10^{13} \mathrm{~g}$, we find a mass-loss rate of $2.2 \times 10^{-15} M_{\odot} \mathrm{yr}^{-1}$, roughly $10 \%$ of the solar wind mass-loss rate.

\subsection{T Tauri Star Case}

In Section 3.1, we demonstrated that the observed distribution of solar flares, when converted to CME masses via our empirical relation between flare energy and CME mass (Equation (2)), correctly recovers the observed distribution of solar CMEs. We moreover developed an analytical representation of the solar flare distribution in an attempt to account for observational biases in the observed solar flare distribution, and hence in our flare-inferred solar CME mass distribution. In this section, we follow the same procedure, now applied to PMS stellar flares in order to arrive at a PMS stellar CME mass-loss rate.

\subsubsection{Empirically Determined TTS CME Mass-loss Rate}

As in the solar case, we can infer an empirical CME massloss rate for TTSs by combining our solar-calibrated flare energy/CME mass relationship extended into the TTS flare regime (Equation (2) and Figure 1) with an empirical flareenergy distribution function for TTS flares.

We utilize the energy distribution of TTS flare rates already measured for the COUP sample by Albacete Colombo et al. (2007). Next, we convert the cumulative distribution of Albacete Colombo et al. (2007) to $d N / d E$ (Figure 3, left panel). Proceeding as in the solar case (Section 3.1), this $d N / d E$ and the CME mass/flare energy relationship (Equation (2) and Figure 1) are used to calculate a CME mass distribution (Section 3.1.2; Figure 3, middle and right panels). Directly summing this inferred CME mass distribution, we obtain a CME mass-loss rate of $\dot{M}_{\mathrm{CME}}=\sum d N / d M M=6.2 \times 10^{-13} M_{\odot} \mathrm{yr}^{-1}$. This is an order of magnitude more mass-loss than the present-day solar wind. As in the solar case, this likely represents a lower limit on the true TTS CME mass-loss rate due to observational biases against the detection of the smallest flares/CMEs.

\subsubsection{Analytically Determined TTS CME Mass-loss Rates}

As in the solar case, the empirical flare-inferred $\dot{M}_{\mathrm{CME}}$ presented above is subject to one clearly problematic bias: the $\mathrm{X}$-ray flare detection limit. Below this limit, $E_{\text {cut }}$, as in the solar case, the flare rate distribution likely continues to increase to ever lower flare energies; if this behavior is as on the Sun, then the distribution likely follows a power law over many dex in energy. The empirical $\dot{M}_{\mathrm{CME}}$ above, then, provides a lower limit for the mass loss. Here, we present an analytical solution that represents an upper limit by attempting to account for the underdetected, lower energy/lower mass flare/CME events.

The power-law fit to the COUP flare energy/event rate distribution above the completeness limit of $E_{\text {cut }}=10^{35.6} \mathrm{erg}$ is 
as described in Equation (3), where $\alpha=2.1$ (Albacete Colombo et al. 2007). We do not have directly observed bounds on TTS CME masses, so we instead perform our distribution integration over flare energy and using our relation between flare energy and CME mass (Equation (2)). The mass-loss rate is then (equivalent to Equation (5), with substitutions):

$$
\begin{aligned}
\dot{M}_{\mathrm{CME}} & =\int_{E_{\min }}^{E_{\max }} \frac{d N}{d E} M_{\mathrm{i}} d E \\
& =\frac{K_{\mathrm{E}} K_{\mathrm{M}}}{(\beta-\alpha+1)}\left(E_{\max }^{\beta-\alpha+1}-E_{\min }^{\beta-\alpha+1}\right) .
\end{aligned}
$$

To place a lower limit on the value of $E_{\min }$ (and thus an upper limit on $\dot{M}_{\mathrm{CME}}$ ), we find the energy at which the X-ray luminosity in flares $\left(L_{\text {flare }}\right)$ is equal to the total stellar X-ray luminosity, $L_{X}$ :

$$
L_{\text {flare }}=\int_{E_{\min }}^{E_{\max }} \frac{d N}{d E} E d E=\frac{K_{\mathrm{E}}}{2-\alpha}\left(E_{\max }^{2-\alpha}-E_{\min }^{2-\alpha}\right)=L_{X} .
$$

For the stellar $L_{X}$, we take a fiducial value of $2.5 \times 10^{30} \mathrm{erg} \mathrm{s}^{-1}$ from the empirical relationship of Preibisch et al. (2005) for a $1 M_{\odot}$ star. We note that the scatter in $L_{\mathrm{X}}$ is rather large (see Figure 3 of Preibisch et al. 2005); the $1 \sigma$ scatter about their linear regression fit to $L_{X}$ as a function of mass is $0.65 \mathrm{dex}$. Thus, our choice of fiducial $L_{X}$ could be as low as $5 \times 10^{29} \mathrm{erg} \mathrm{s}^{-1}$ or as high as $1 \times 10^{31} \mathrm{erg} \mathrm{s}^{-1}$. We choose the maximum energy in Equation (11) to simply be the most energetic flare observed in the COUP sample, $\sim 10^{37} \mathrm{erg}$. Evaluating Equation (11), we find $E_{\min } \gtrsim 3.4 \times 10^{29} \mathrm{erg}$. With these parameters, we find an upper limit to the mass-loss rate for TTS via Equation (9): $\dot{M}_{\mathrm{CME}} \lesssim$ $3.2 \times 10^{-10} M_{\odot} \mathrm{yr}^{-1}$.

This mass-loss rate is most sensitive to the flare frequency/ energy distribution slope $\alpha$; were we to vary $\alpha$ within the reported uncertainty, the mass-loss rates would change by a couple orders of magnitude: for $\alpha$ values of 2.05 and 2.2, $\dot{M}_{\mathrm{CME}}$ is $1.9 \times 10^{-9} M_{\odot} \mathrm{yr}^{-1}$ and $7.1 \times 10^{-11} M_{\odot} \mathrm{yr}^{-1}$, respectively. This is consistent with the solar case and our expectation that the CME mass-loss rates would not exceed typical wind mass-loss rates for young stars.

\subsection{CME Mass-loss Rates Compared to Steady Wind, Observed Outflow Rates}

We have devised a method to infer the CME mass-loss rate from the observed flaring rate together with our empirical relation between flare energy and CME mass. Testing this method on the Sun, our empirical method predicts the CME mass-loss rate within less than a percent of the observed value. We then applied the same method using an analytical powerlaw representation of the observed flare distribution in order to attempt to account for observational biases against the lowestenergy flares (and thus against the lowest mass CMEs). This analytical method predicts 2.8 times more mass loss than is observed. With respect to the solar wind mass-loss rate $\left(10^{-14} M_{\odot} \mathrm{yr}^{-1}\right.$, Li 1999), the empirical result (as well as the observed CME mass-loss rate) is $4 \%$ of the wind mass-loss rate, while the analytical result is that CMEs lose mass at $10 \%$ the solar wind rate. Given that the observed CME mass-loss rate is itself underpredicted (only half of the CMEs reported in the database have measured masses), and the observational bias against the lowest mass flares is present in our empirical result as well as the actual solar data, we would anticipate the analytic calculation to be greater than the observed mass lost. As such, these two approaches-empirical and analytical-serve as lower and upper limits, respectively.

In the TTS case, our empirically calculated lower limit on the stellar CME mass-loss rate is $\sim 6 \times 10^{-13} M_{\odot} \mathrm{yr}^{-1}$. Our analytically derived mass-loss rates range from $\sim 10^{-11}-10^{-9} M_{\odot} \mathrm{yr}^{-1}$ (when taking into account the error on the stellar flare frequency power-law slope). For comparison, TTS wind mass-loss rates have been derived from various line diagnostics: for TW Hya, Dupree et al. (2005) report $\dot{M}_{\mathrm{O} \text { VII }} / \phi=2.3 \times 10^{-11} M_{\odot} \mathrm{yr}^{-1}$ and $\dot{M}_{\mathrm{C} \text { III }} / \phi=1.3 \times 10^{-12} M_{\odot} \mathrm{yr}^{-1}$, where $\phi$ is the fractional stellar surface area from which the wind originates (e.g., a more collimated, polar wind will have $\phi \lesssim 0.3$ ). These outflow rates are less than the $\mathrm{H} \alpha$ emission derived accretion rate for TW Hya of $4 \times 10^{-10} M_{\odot} \mathrm{yr}^{-1}$ (Muzerolle et al. 2000). In cases of other accreting systems, wind mass-loss rates inferred from accretion signatures in spectral lines range from $10^{-9}$ to $10^{-7}$ (Hartigan et al. 1995).

\section{ANGULAR MOMENTUM LOSS VIA CMEs}

Having calculated mass-loss rates from PMS stellar CMEs, we now focus on whether or how these episodic mass-loss events could impact the angular momentum evolution of a TTS. We have determined order-of-magnitude CME mass-loss rates, calculated empirically as well as analytically, giving approximate lower and upper limits of $\sim 10^{-12}$ and $\sim 10^{-9} M_{\odot} \mathrm{yr}^{-1}$, respectively (Sections 3.2.1 and 3.2.2). In this section, we estimate magnetic lever arm lengths to calculate torques and assess their relative importance in spin evolution.

\subsection{What is the Magnetic Lever Arm Length?}

The torque on the star, due to a steady, magnetized wind can be written

$$
T_{\mathrm{w}}=-\dot{M}_{\mathrm{w}} \Omega_{*} r_{\mathrm{A}}^{2}
$$

where $\dot{M}_{\mathrm{w}}$ is the mass-loss rate in a steady wind, $\Omega_{*}$ is the angular rotation rate of the star, and $r_{\mathrm{A}}$ is the average "lever arm" radius in the wind (e.g., Matt \& Pudritz 2008a and references therein).

Computing the lever arm radius precisely requires knowledge of the three-dimensional flow structure and magnetic field configuration (e.g., Mestel 1984). For time-variable flows, such as CMEs, the situation is even more complicated. Thus, here we will only estimate the lever arm radius that is appropriate for an ensemble of CMEs and that is based upon our current understanding of magnetized stellar winds. Based on simulations of steady-state, ideal MHD winds from stars with dipole magnetic field and rotating at $10 \%$ of breakup speed (Matt \& Pudritz 2008a; Matt et al. 2012) found

$$
\frac{r_{\mathrm{A}}}{R_{*}} \approx 2.1\left(\eta \frac{B_{*}^{2} R_{*}^{2}}{\dot{M}_{\mathrm{CME}} v_{\mathrm{esc}}}\right)^{0.22}
$$

where $B_{*}$ is the equatorial field strength of the global/dipolar magnetic field at the stellar surface, $R_{*}$ is the radius of the star, and $v_{\text {esc }}$ is the escape speed from the stellar surface. Here, we have introduced a factor $\eta$, in order to account for the difference in the effective lever arm length for an ensemble of eruptive mass-loss events ( $\left.\dot{M}_{\mathrm{CME}}\right)$, as compared to a steady wind $\left(\dot{M}_{\mathrm{w}}\right)$.

There are several factors that modify the effective lever arm length in an eruptive outflow, compared to a steady one. 
The first is that the mass loss is not continuous, but happens in discrete bursts/ejections. For simplicity, we consider that the mass loss occurs with an average rate of $\dot{M}_{\mathrm{w}}=\dot{M}_{\mathrm{CME}}$, but occurring via discrete bursts that happen for a fraction $f_{t}$ of the time $\left(f_{t}<1\right)$. Thus, each burst has an instantaneous mass-loss rate of $f_{t}^{-1} \dot{M}_{\mathrm{CME}}$, and the factor of $\eta$ in Equation (13) should include a factor of $f_{t}$, in order to take this into account. It is clear that a time-dependent wind is less efficient at extracting angular momentum than a steady wind with the same average mass-loss rate. In the inferred distribution of CMEs (see Figure 3, middle panel), the mass loss is dominated by the lowest energy events, of which there are $\sim 10$ per year. To estimate $f_{t}$, we also need to specify the duration of each event-i.e., the duration over which we can think of the CME as a steady wind from the surface of the star. As an order-of-magnitude estimate, we consider the time for a CME traveling several hundred $\mathrm{km} \mathrm{s}^{-1}$ to cross several stellar radii, which is of the order of one hour. For 10 events per year with a duration of one hour, $f_{t} \sim 10^{-3}$. To calculate our upper limit to $\dot{M}_{\mathrm{CME}}$ in Section 3.2.2, we considered that the flare rate distribution extends to much lower energies than observed. In this case, the event frequencies of the lowest energy flares are much higher, implying a larger value for $f_{t}$.

The second factor modifying the effective lever arm length in an eruptive flow is that the CME events are not globally distributed in the corona, but take place over a fraction of the total solid angle of whole sky. More importantly for the angular momentum flow, not all of the stellar magnetic flux participates in the azimuthal acceleration of the outflow. To take this into account, the factor $\eta$ in Equation (13) should include a factor of $f_{\psi}^{2}$, where $f_{\psi}$ is the fraction of (unsigned) magnetic flux participating in the CME flow, compared to the total stellar surface magnetic flux associated with the global/dipole field. The unsigned flux participating in a CME should approximately equal the geometric area times the field strength of the underlying active region. Generally speaking, and almost by definition, the magnetic field strengths of active regions are much stronger than the global field strength, while the areas are much smaller than the total stellar surface area. The product of these two approximates $f_{\psi}$, and it is not clear in general whether this will be very different from unity. For the purpose of this section, we assume $f_{\psi} \sim 0.1-1.0$.

Other factors that influence the effective lever arm length are the angle that the direction of the CME flow makes with respect to the rotation axis of the star and that the acceleration of the CMEs may be significantly different than the acceleration of the steady wind in the simulations of Matt et al. Given the much larger uncertainties discussed above, we neglect these effects for the present analysis and approximate $\eta=f_{t} f_{\psi}^{2}$. We consider here a range of $10^{-3} \leqslant \eta \leqslant 1$, but acknowledge that an even wider range may be possible. Note that the case of $\eta=1$ is equivalent to the case of a steady wind.

To compute the lever arm length as a function of time, we use a model for the evolution of a $1 M_{\odot}$ star from Siess et al. (2000), which gives the stellar radius $R_{*}$ (and thus surface escape speed $v_{\text {esc }}$ ) as a function of stellar age. The upper left panel of Figure 4 shows the evolution of $R_{*}$. To compute the lever arm length, we must specify the surface magnetic field strength, mass-loss rate, and $\eta$. For simplicity, we adopt a singular value of $B_{*}=600 \mathrm{G}$ (corresponding to the equatorial field strength of the dipole field measured on BP Tau; Donati et al. 2008), and assume that it is constant in time. The range of possible values of $B_{*}$ for TTSs, as well as how this may evolve in time, is not well constrained observationally, which introduces an uncertainty in our calculated values. The upper right panel of Figure 4 shows the Alfvén radius as a function of time, for four combinations of the extreme values of mass-loss rate and $\eta$ discussed above. In the figure, the vertical triple-dot-dashed lines mark the approximate age range of TTSs (for which this calculation is valid), corresponding roughly to an age of a few Myr, plus or minus a few Myr. Since the mass-loss rates we are considering correspond to TTSs, the calculation is only valid/relevant between these two vertical lines, but we show a wider range of ages for context/illustrative purposes. It is clear that the range of $\eta$ and $\dot{M}_{\mathrm{CME}}$ considered corresponds to a range of a factor of about 13 in the possible Alfvén radii at any given age. In the age range of TTSs, the Alfvén radius could be in the range of 3-70 $R_{*}$, depending on the wind parameters.

Observationally, the largest magnetic structures are seen to exist at distance scales comparable to Alfvén radii calculated here: the magnetic loops of Favata et al. (2005) were inferred to be from 0.1 to $55 R_{*}$ in extent (assuming that these were indeed single loops), and cool prominences have been seen to extend up to a few stellar radii from TTS (Massi et al. 2008; Skelly et al. 2008). At later evolutionary stages, solar type stars are still seen to have extended, suspended material: the $50 \mathrm{Myr}$ old (Luhman et al. 2005) solar analog AB Dor was observed to have cool, corotating clouds at distances from $\sim 9$ to $20 R_{*}$ from the rotation axis, and were estimated to have masses $\gtrsim 10^{18} \mathrm{~g}$ (Collier Cameron \& Robinson 1989). Dunstone et al. (2006) observed prominences on Speedy Mic, a 30 Myr old K3 dwarf, and estimated masses to be $\sim 2 \times 10^{17} \mathrm{~g}$. In stellar outflows, the Alfvén radii are typically comparable to or slightly larger than the size of the largest closed magnetic loops (e.g., Mestel \& Spruit 1987). Thus, the measured sizes of post-flare loops and prominences may represent an approximate lower limit on the Alfvén radii for these systems.

\subsection{How Important is the Torque?}

The spin rate of stars will change in response to external torques and changes in the structure of the star (and subsequent change in the stellar moment of inertia). The conservation of angular momentum for an isolated star rotating as a solid body can be expressed as

$$
I_{*} \frac{d \Omega_{*}}{d t}=T_{\mathrm{w}}-\Omega_{*} \frac{d I_{*}}{d t},
$$

where $I_{*} \equiv k^{2} M_{*} R_{*}^{2}$ is the moment of inertia of the star, $\Omega_{*}$ is the angular spin rate of the star, and $T_{w}$ is the wind torque (Equation (12)); here, we are treating the CME outflow like a wind, so $T_{\mathrm{w}}=T_{\mathrm{CME}}$ ), and $k^{2}$ is the normalized radius of gyration (defined by the radial mass distribution). For a star in a tight binary system or a star that is still actively accreting from a disk, there will be additional torque terms in Equation (14), which we neglect here in order to isolate the effects of CMEs alone.

To compute the relative strength of the terms in this equation, we use the $1 M_{\odot}$ Siess et al. (2000) model to specify the evolution of $k^{2}$ and $R_{*}$. In this and in most other PMS stellar evolution models, the star is fully or nearly fully convective during the first $\sim 10 \mathrm{Myr}$ of evolution. In convective regions, the mixing of material redistributes angular momentum on a timescale that is much shorter than evolutionary timescales. Thus, external torques effectively act upon the entire mass of the convection zone; for a fully convective PMS star, the external torques act on the entire star (as opposed to acting only on 

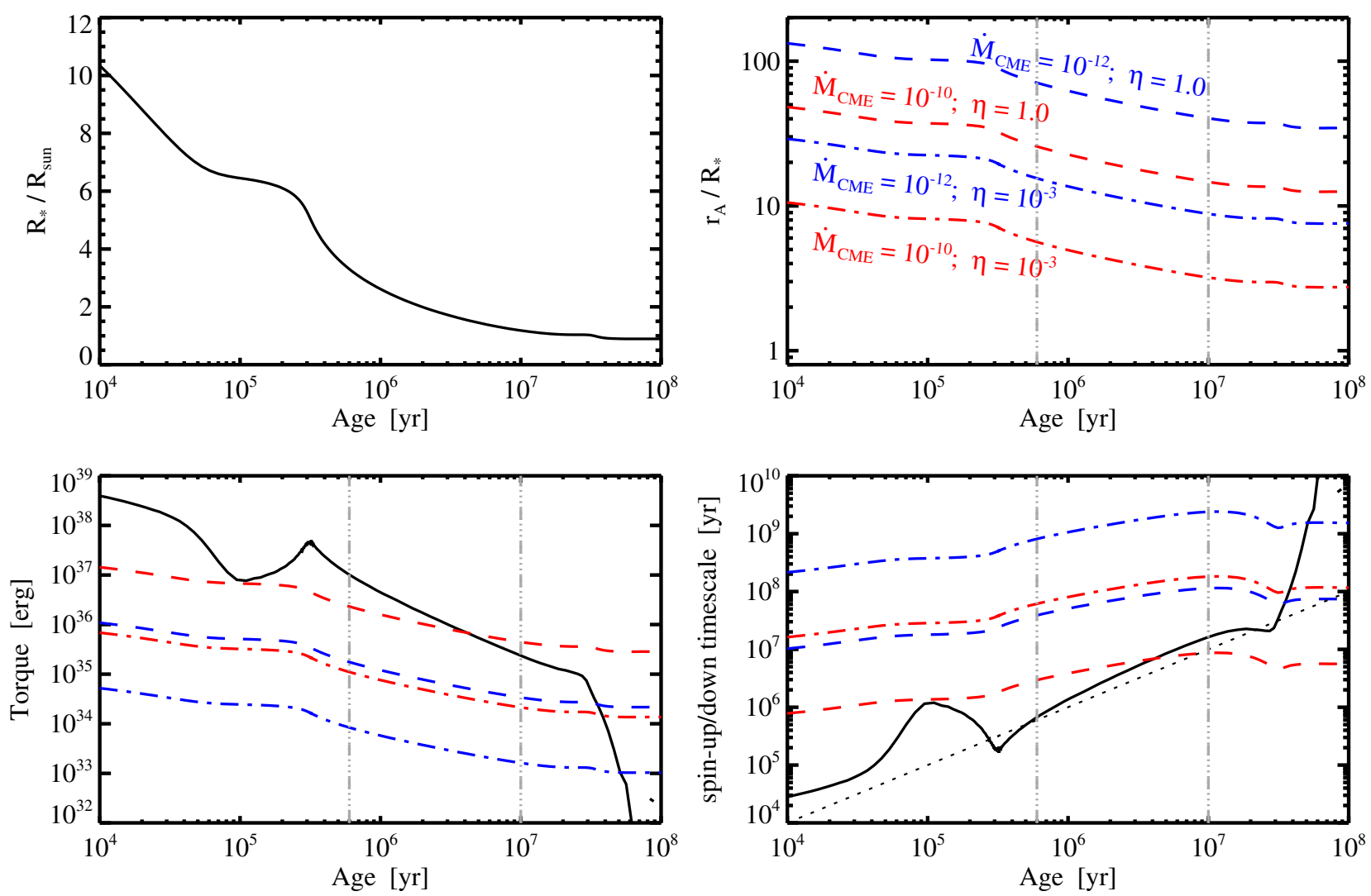

Figure 4. Upper left panel: evolution of the photospheric radius of a one solar mass star, as a function of age, from the model tracks of Siess et al. (2000). Upper right panel: effective magnetic lever arm (Alfvén) radius in CME outflows from the same star, with a magnetic field strength of $600 \mathrm{G}$ and four different combinations of mass-loss rate $\left(\dot{M}_{\mathrm{CME}}\right)$ and efficiency factor $(\eta)$, as indicated on the plot $\left(\dot{M}_{\mathrm{CME}}\right.$ is given in units of $\left.M_{\odot} \mathrm{yr}^{-1}\right)$. The vertical triple-dot-dashed lines show the approximate age range of the T Tauri phase. Lower left panel: the solid line shows the "pseudo-torque," which shows the contribution to the evolution of stellar rotation rate that is due to the contraction and changes of internal structure of the star (see the text). The colored lines show the CME-outflow torque, which acts to spin down the star, corresponding to different outflow parameters (line styles correspond to the upper right panel). Lower right panel: the solid line shows the spin-up timescale, due only to the contraction of changes of internal structure of the star, as a function of stellar age. The colored lines show the spin-down timescale due to CME outflows, corresponding to different outflow parameters (corresponding to the upper right panel). The dashed line corresponds to a spin-timescale equal to the age of the star.

(A color version of this figure is available in the online journal.)

a thin shell that is rotationally decoupled from the interior). There have been observations of possible differential rotation in a few TTSs, with amplitudes of $\sim 10 \%$ of the bulk/average rotation rate (e.g., Herbst et al. 2006), but in the vast majority of TTSs studied no measurable differential rotation is observed. Thus, here we follow convention and treat the star as a solidbody rotator, where $\Omega_{*}$ represents the bulk rotation rate, and the error associated with this approximation is much smaller than for other unknowns (such as the value of $\eta$ ). In order to specify $\Omega_{*}$, we simply assume that the star is always rotating at a fraction $f=10 \%$ of breakup speed, in order to approximately represent the "slow rotators" in observed TTS spin distributions (e.g., Herbst et al. 2007). This means that the rotation period is assumed to be

$$
P_{*}=\frac{2 \pi}{\Omega_{*}} \approx 1.17 \text { days }\left(\frac{0.1}{f}\right)\left(\frac{R_{*}}{R_{\odot}}\right)^{3 / 2}\left(\frac{M_{\odot}}{M_{*}}\right)^{1 / 2} .
$$

Since both terms on the right-hand side of Equation (14) are proportional to $\Omega_{*}$, the relative value of these two terms is independent of the assumed value of $\Omega_{*}$.

The bottom left panel of Figure 4 compares the two terms on the right-hand side of Equation (14) as a function of time. The broken lines show the value of $-T_{\mathrm{CME}}$, where we have multiplied by -1 in order to compare the absolute value of the terms on a logarithmic scale. The different line styles and colors correspond to the assumptions and range of possible values of stellar wind parameters discussed in the previous section and labeled in the top right panel of the figure. The stellar wind torque always acts to spin down the star (decrease $\Omega_{*}$ ). The solid line in the bottom left panel shows the last term on the right-hand side of Equation (14). This term has the dimensions of torque and can be thought of as a pseudo torque, which describes how the spin rate of the star changes due to changes in the moment of inertia (i.e., even if total angular momentum were conserved), and which acts to spin up the star (increase $\Omega_{*}$ ).

In order to assess how the terms in Equation (14) may affect the stellar spin rate, it is useful to compute a spin-up or spindown timescale by dividing the total angular momentum by the torque. By rearranging Equation (14), the spin-up/down time can be written

$$
\frac{\Omega_{*}}{\dot{\Omega}_{*}}=\frac{\Omega_{*}}{T_{\mathrm{CME}} / I_{*}-\Omega_{*} / I_{*}\left(d I_{*} / d t\right)} .
$$

When the CME-outflow torque dominates, the star will spin down on a timescale

$$
\tau_{\mathrm{CME}}=\frac{I_{*} \Omega_{*}}{T_{\mathrm{CME}}}=k^{2}\left(\frac{M_{*}}{\dot{M}_{\mathrm{CME}}}\right)\left(\frac{R_{*}}{r_{\mathrm{A}}}\right)^{2} .
$$


By contrast, when the changes in stellar structure dominate, the star will spin up on a timescale

$$
\tau_{\text {struct }}=-\frac{I_{*}}{\left(d I_{*} / d t\right)} .
$$

One advantage of looking at things in this way is that it is independent of the current spin rate of the star. In the bottom right panel of Figure 4 , we show $\tau_{\text {struct }}$ (solid line) and $\tau_{\mathrm{CME}}$ (broken lines, corresponding to the various wind parameters indicated in the upper right panel), as a function of stellar age, for the $1 M_{\odot}$ Siess et al. model. At any given time, the shortest timescale is the dominant one, and if it is comparable to or shorter than the age of the star (shown as a dotted line), then it will have a noticeable effect on the stellar spin rate.

It is clear from Figure 4 that the stellar contraction is expected to effectively spin the star up during the first $30 \mathrm{Myr}$ (in the absence of any external torques). The figure shows that this pseudo-torque from stellar contraction completely dominates over the angular momentum loss associated with CMEs, for most of the range of parameters considered here. The only exception is that for the highest mass outflow rates allowable by our analysis, and for $\eta \sim 1$, the angular momentum loss by CMEs becomes comparable to the contraction pseudo-torque at an age of a few million years and dominates it thereafter.

Many of the stars in the COUP sample are actively accreting from a surrounding accretion disk. It is therefore interesting to consider the angular momentum exchange between the star and disk, arising from this interaction. Matt \& Pudritz (2008b) demonstrated that the spin-down torque associated with the magnetic connection between the star and disk will be less than the torque from a stellar outflow, in the case where the Alfvén radius is not too large and when the magnetic coupling to the disk is strong. Using their "preferred" values for the coupling, they demonstrated that a stellar outflow will carry away more angular momentum than the star-disk interaction, as long as $r_{\mathrm{A}}<84 R_{*}$. As demonstrated in the upper right panel of Figure $4, r_{\mathrm{A}} \leqslant 70 R_{*}$ for all of the outflow parameters considered here, so the spindown torque due to a star-disk magnetic connection should be negligible. ${ }^{5}$ Accreting stars can also experience strong spin-up torques, mainly associated with the accretion of high specific angular momentum from nearly-Keplerian disks. The spin-up torque from accretion is given by $\dot{M}_{\mathrm{a}} \sqrt{G M_{*} R_{\mathrm{t}}}$, where $\dot{M}_{\mathrm{a}}$ is the accretion rate, and $R_{\mathrm{t}}$ is the radial location of the inner truncation of disk (e.g., Ghosh \& Lamb 1978; Matt \& Pudritz 2005b). As an example, for the star considered here, accreting at a rate of $10^{-8} M_{\odot} \mathrm{yr}^{-1}$, with a truncation radius of a few stellar radii, and at an age of $6 \times 10^{5}$ years (values appropriate for the early TTS phase), the resulting accretion torque is $\sim 10^{37} \mathrm{erg}$. This spin-up torque is comparable to the contraction pseudo-torque at the same age (lower left panel of Figure 4).

Thus, depending on the stellar age, accretion rate, etc., the processes acting to spin up the star may be dominated by either contraction or accretion. In order to counteract this spin-up torque and to explain the observations of slow rotators at all TTS ages, the mass outflow rates must be high, the magnetic field strengths must be large, and/or additional spin-down torques (that are not considered here) must be present. The CME outflows inferred in the present work could be important near the end of the TTS phase, in the case of weakly- or nonaccreting stars, and if the mass-loss rate is near our upper limit $\left(\dot{M}_{\mathrm{CME}} \gtrsim 10^{-10} M_{\odot} \mathrm{yr}^{-1}\right)$ and $\eta \sim 1$.

\footnotetext{
5 The analysis of Matt \& Pudritz (2008b) is valid only for $\eta=1$. It could be modified to consider $\eta \neq 1$, but the overall results are not affected.
}

\section{DISCUSSION}

In this work, we derive mass-loss rates for CMEs from solar type PMS stars. This is a first attempt to estimate PMS mass loss via this mechanism and to calibrate it explicitly against the Sun and against observations of flaring magnetic loops on PMS stars. We find the total CME mass-loss rate to be in the range of $10^{-12}$ to $10^{-9} M_{\odot} \mathrm{yr}^{-1}$. This is relatively modest by comparison with mass-loss rates observed for TTS winds. However, the magnetic lever arms associated with the largest PMS CMEs, as inferred from the COUP sample stars and scaled from the Sun, are large - up to tens of stellar radii-so the resulting torques can be significant. Assuming that the CME mass-loss rate for a typical $\sim 1 M_{\odot}$ TTS is $\lesssim 10^{-10} M_{\odot} \mathrm{yr}^{-1}$, the associated spindown torque is likely too small to counteract the spin-up effects of contraction and accretion in the T Tauri phase. However, the spin-down torque from CMEs could be important after an age of a few Myr if the mass-loss rate is $\gtrsim 10^{-10} M_{\odot} \mathrm{yr}^{-1}$, the accretion rate is low, and the angular momentum loss is efficient. Our estimates of the angular momentum loss efficiency (i.e., the magnetic lever arm radii; see Section 4) are based upon axisymmetric, steady-state wind calculations, and three-dimensional simulations are needed to improve these estimates.

Our stellar CME model is derived from relating solar activity to stellar; in one sense, we immediately underestimate mass losses via CMEs by only selecting solar CMEs that occurred with flares. We find, however, that much of the mass loss comes from the CMEs associated with flares. The most massive CMEs are associated with the most powerful flares, and the most energetic flares are those most often associated with CMEs (Aarnio et al. 2011). Thus, using stellar flare activity to determine a stellar CME rate likely represents a lower bound on the stellar CME activity.

One of the most pivotal parts of this calculation is the extrapolation of the CME mass/flare energy relationship. The adopted direct extrapolation, while crossing many orders of magnitude, is indeed physically motivated, and introduces the fewest new assumptions. We are able to, using the COUP "superflare" observations, justify the extrapolation up to TTS flare energies; our premise is that these loops represent proxies for stellar CME masses as the loops exist within the density and height regimes from which CMEs are generally launched on the Sun. The consistency with the solar CME mass/flare energy relationship is particularly compelling given that it spans 6 dex in flare energy.

In adopting the stellar X-ray flare frequency relationship of Albacete Colombo et al. (2007), we have generated an "ensemble mass-loss rate"; in doing this, we have neglected to separate out any mass-dependent characteristics of the distribution. There would be, however, significant differences in the flare activity for stars of the same mass with different rotation rates. Given the mixed sample of classical and weak-line TTS in the COUP, there are also self-absorption effects (by the accretion columns, Gregory et al. 2007, or a circumstellar disk inclined to our line of sight) that could introduce bias into a flare frequency distribution. It was our intent in using the COUP sample to diminish all of these observational effects via the large number of stars observed in various activity and evolutionary phases, as well as inclinations and rotation rates. Future work could include analyzing subsets of the COUP data set to see whether there is an X-ray luminosity dependence on the flare rate (and thus, a difference in CME mass-loss rates as well). 
As noted in Section 3.2.2, one particular difficulty with this analysis was the sensitivity of our analytical solutions to the value of the power-law slope. Within the errors on these quantities, we find our mass-loss rates derived analytically could span two orders of magnitude. The empirically derived massloss rates, while derived from incomplete samples, represent robust lower limits. In the solar case, our analytical mass-loss rate did predict more mass loss than was observed in the LASCO database, but as discussed in Section 2, the LASCO database itself is missing many CME masses, so the total mass-loss rate is undoubtedly higher. Despite this apparent overprediction, we still derive a mass-loss rate below the steady solar wind massloss rate, which is as anticipated.

Observing stellar CMEs would be ideal for addressing the most uncertain parameters in this work, but presently, understanding the observational signatures and being able to detect a stellar CME remain outstanding problems. Efforts have been made with EUV spectroscopy; Leitzinger et al. (2011) point out a lack of simultaneous solar spectra during flare/CME events, creating great difficulty for the interpretation of stellar data. Additionally, managing to catch a stellar CME with the right set of parameters for observation (e.g., density and projection) requires sufficient observing time to increase the probability of detection. For a steady, hot (1 MK), coronal wind, Matt \& Pudritz (2007) calculate a mass-loss rate of $1 \times 10^{-9} M_{\odot} \mathrm{yr}^{-1}$ would produce strong EUV and X-ray emission, much higher than is observed. Our highest mass-loss rate estimate, $\sim 10^{-9} M_{\odot} \mathrm{yr}^{-1}$, is probably unlikely given the strong observational signature that would attend such a mass-loss rate. Interestingly, however, the authors also found that decreasing the mass-loss rate by a factor of 10 decreased the excess emission by a factor of 100 . Given the "bursty" nature of our CMEs, and the likely high cadence of these bursts, and potentially cooler temperatures (solar CMEs are observed to rapidly expand as they travel away from the Sun), a significant level of mass loss could easily escape detection.

The COUP "superflaring" targets were generally weak lined TTS (Aarnio et al. 2010); it is likely that accretors could show an enhanced activity rate and thus more frequent CMEs, as well as accretion-driven winds (Matt \& Pudritz 2008a) which could further deplete angular momentum. The presence of a close-in disk in accreting systems might mitigate the enhanced CME rate with reduced lever arm lengths, however modeling work (Orlando et al. 2011) has shown star-disk flaring can disrupt circumstellar disk material, causing a magnetorotational instability that then generates an accretion flow. Thus, in a cyclical fashion, large scale flaring can lead to accretion, the accretion then powering winds, outflows, and more reconnection, resulting in further mass/angular momentum loss. Cranmer (2009) illustrate one mechanism by which accretion could power a wind: infalling material impacting the stellar surface would generate Alfvén waves, the propagation of which could trigger reconnection, with accretion thus powering activity.

\section{SUMMARY AND CONCLUSIONS}

This represents a preliminary effort at calculating mass and angular momentum losses for T Tauri stars via scaled-up, solaranalog coronal mass ejections. Our calculations are based on well-observed flares among a large sample of solar-type PMS stars, and our analysis methods have been tested for the most well-understood case, the Sun. Beginning with an established relationship between solar flare energies and CME masses (Aarnio et al. 2011), we use the observed flare frequency distribution to infer a CME mass distribution. We are able to infer empirically and analytically solar CME mass-loss rates that are consistent with observations, and also consistent with expectations for a mass-loss rate once observational biases are carefully accounted for.

Our lower limit on the mass shed via CMEs in a generic TTS case is $6.2 \times 10^{-13} M_{\odot} \mathrm{yr}^{-1}$. To obtain an upper limit on the stellar CME mass-loss rate, we calculate analytic solutions to account for observational biases (most importantly, the stellar $\mathrm{X}$-ray flare detection limit); our upper limit to the mass-loss rate via CMEs is $\dot{M}_{\mathrm{CME}} \lesssim 2 \times 10^{-9} M_{\odot} \mathrm{yr}^{-1}$.

Finally, we assess the resulting torque against the star's rotation provided by the CME mass loss. We find that within the ranges of mass-loss rates and effective lever arm lengths (reflecting the difference in steady versus "bursty" mass loss) being considered, the resulting Alfvén radii would span a large range, 3-70 $R_{*}$, consistent with previous inferences of the physical sizes of large-scale magnetic structures. We find that near our upper limit mass-loss case $\left(\gtrsim 10^{-10} M_{\odot} \mathrm{yr}^{-1}\right)$, this mechanism could be effective for slowing stellar rotation on a timescale comparable to TTS lifetimes.

We acknowledge NSF grant AST-0808072 (K. Stassun, PI).

\section{REFERENCES}

Aarnio, A., Llama, J., Jardine, M., \& Gregory, S. G. 2012, MNRAS, 421, 1797 Aarnio, A. N., Stassun, K. G., Hughes, W. J., \& McGregor, S. L. 2011, Sol. Phys., 268,195

Aarnio, A. N., Stassun, K. G., \& Matt, S. P. 2009, in AIP Conf. Ser. 1094, Cool Stars, Stellar Systems and the Sun, ed. E. Stempels (Melville, NY: AIP), 337 Aarnio, A. N., Stassun, K. G., \& Matt, S. P. 2010, ApJ, 717, 93

Albacete Colombo, J. F., Caramazza, M., Flaccomio, E., Micela, G., \& Sciortino, S. 2007, A\&A, 474, 495

Andrews, M. D. 2003, Sol. Phys., 218, 261

Audard, M., Briggs, K. R., Grosso, N., et al. 2007, A\&A, 468, 379

Christe, S., Hannah, I. G., Krucker, S., McTiernan, J., \& Lin, R. P. 2008, ApJ, 677,1385

Collier Cameron, A., \& Robinson, R. D. 1989, MNRAS, 238, 657

Cranmer, S. R. 2008, ApJ, 689, 316

Cranmer, S. R. 2009, ApJ, 706, 824

Donati, J.-F., Jardine, M. M., Gregory, S. G., et al. 2008, MNRAS, 386, 1234

Dunstone, N. J., Collier Cameron, A., Barnes, J. R., \& Jardine, M. 2006, MNRAS, 373, 1308

Dupree, A. K., Brickhouse, N. S., Smith, G. H., \& Strader, J. 2005, ApJ, 625, L131

Edwards, S., Fischer, W., Hillenbrand, L., \& Kwan, J. 2006, ApJ, 646, 319

Edwards, S., Fischer, W., Kwan, J., Hillenbrand, L., \& Dupree, A. K. 2003, ApJ, 599, L41

Favata, F., Flaccomio, E., Reale, F., et al. 2005, ApJS, 160, 469

Feigelson, E., Townsley, L., Güdel, M., \& Stassun, K. 2007, in Protostars and Planets V, ed. B. Reipurth, D. Jewitt, \& K. Keil (Tucson, AZ: Univ. Arizona Press), 313

Fernandez, M., Ortiz, E., Eiroa, C., \& Miranda, L. F. 1995, A\&AS, 114, 439

Getman, K. V., Feigelson, E. D., Grosso, N., et al. 2005, ApJS, 160, 353

Getman, K. V., Feigelson, E. D., Micela, G., et al. 2008, ApJ, 688, 437

Ghosh, P., \& Lamb, F. K. 1978, ApJ, 223, L83

Gopalswamy, N., Yashiro, S., Michalek, G., et al. 2009, Earth Moon Planets, 104,295

Gregory, S. G., Wood, K., \& Jardine, M. 2007, MNRAS, 379, L35

Güdel, M. 2008, Astron. Nachr., 329, 218

Guedel, M., Guinan, E. F., \& Skinner, S. L. 1997, ApJ, 483, 947

Haisch, K. E., Jr., Lada, E. A., \& Lada, C. J. 2001, ApJ, 553, L153

Hamaguchi, K., Grosso, N., Kastner, J. H., et al. 2012, ApJ, 754, 32

Hartigan, P., Edwards, S., \& Ghandour, L. 1995, ApJ, 452, 736

Hayashi, M. R., Shibata, K., \& Matsumoto, R. 1996, ApJ, 468, L37

Herbst, W., Dhital, S., Francis, A., et al. 2006, PASP, 118, 828

Herbst, W., Eislöffel, J., Mundt, R., \& Scholz, A. 2007, in Protostars and Planets V, ed. B. Reipurth, D. Jewitt, \& K. Keil (Tucson, AZ: Univ. Arizona Press), 297

Herbst, W., Herbst, D. K., Grossman, E. J., \& Weinstein, D. 1994, AJ, 108, 1906 
Hudson, H. S. 1991, Sol. Phys., 133, 357

Johns-Krull, C. M. 2007, ApJ, 664, 975

Johns-Krull, C. M., Valenti, J. A., \& Saar, S. H. 2004, ApJ, 617, 1204

Johnstone, C. P., Gregory, S. G., Jardine, M. M., \& Getman, K. V. 2012, MNRAS, 419, 29

Kastner, J. H., Richmond, M., Grosso, N., et al. 2004, Nature, 430, 429

Kwan, J., Edwards, S., \& Fischer, W. 2007, ApJ, 657, 897

Leitzinger, M., Odert, P., Ribas, I., et al. 2011, A\&A, 536, A62

Li, J. 1999, MNRAS, 302, 203

Luhman, K. L., Stauffer, J. R., \& Mamajek, E. E. 2005, ApJ, 628, L69

Massi, M., Ros, E., Menten, K. M., et al. 2008, A\&A, 480, 489

Matt, S., \& Pudritz, R. E. 2005a, ApJ, 632, L135

Matt, S., \& Pudritz, R. E. 2005b, MNRAS, 356, 167

Matt, S., \& Pudritz, R. E. 2007, in IAU Symp. 243, Star-Disk Interaction in Young Stars, ed. J. Bouvier \& I. Appenzeller (Cambridge: Cambridge Univ. Press), 299

Matt, S., \& Pudritz, R. E. 2008a, ApJ, 678, 1109

Matt, S., \& Pudritz, R. E. 2008b, ApJ, 681, 391

Matt, S. P., MacGregor, K. B., Pinsonneault, M. H., \& Greene, T. P. 2012, ApJ, 754, L26

Matt, S. P., Pinzón, G., de la Reza, R., \& Greene, T. P. 2010, ApJ, 714, 989

Mestel, L. 1984, in 3rd Cambridge Workshop on Cool Stars, Stellar Systems, and the Sun, ed. L. H. S. L. Baliunas (Lecture Notes in Physics, Vol. 193; Berlin: Springer), 49

Mestel, L., \& Spruit, H. C. 1987, MNRAS, 226, 57

Muzerolle, J., Calvet, N., Briceño, C., Hartmann, L., \& Hillenbrand, L. 2000, ApJ, 535, L47

Orlando, S., Reale, F., Peres, G., \& Mignone, A. 2011, MNRAS, 415, 3380

Peres, G., Orlando, S., \& Reale, F. 2004, ApJ, 612, 472

Peres, G., Orlando, S., \& Reale, F. 2006, in AIP Conf. Ser. 848, Recent Advances in Astronomy and Astrophysics, ed. N. Solomos (Melville, NY: AIP), 41

Petrov, P. P., Gahm, G. F., Stempels, H. C., Walter, F. M., \& Artemenko, S. A. 2011, A\&A, 535, A6
Preibisch, T., Kim, Y.-C., Favata, F., et al. 2005, ApJS, 160, 401

Reale, F., Betta, R., Peres, G., Serio, S., \& McTiernan, J. 1997, A\&A, 325, 782 Reiners, A., \& Basri, G. 2007, ApJ, 656, 1121

Shu, F., Najita, J., Ostriker, E., et al. 1994, ApJ, 429, 781

Siess, L., Dufour, E., \& Forestini, M. 2000, A\&A, 358, 593

Skelly, M. B., Unruh, Y. C., Cameron, A. C., et al. 2008, MNRAS, 385, 708

Skinner, S. L. 1993, ApJ, 408, 660

Skinner, S. L., Guedel, M., Koyama, K., \& Yamauchi, S. 1997, ApJ, 486, 886

Stassun, K. G., Mathieu, R. D., Mazeh, T., \& Vrba, F. J. 1999, AJ, 117, 2941

Stassun, K. G., van den Berg, M., \& Feigelson, E. 2007, ApJ, 660, 704

Stassun, K. G., van den Berg, M., Feigelson, E., \& Flaccomio, E. 2006, ApJ, 649,914

Stelzer, B., Hubrig, S., Orlando, S., et al. 2009, A\&A, 499, 529

Väänänen, M. K., Schultz, J., \& Nevalainen, J. 2009, Balt. Astron., 18, 233

Valenti, J. A., Basri, G., \& Johns, C. M. 1993, AJ, 106, 2024

Vidotto, A. A., Opher, M., Jatenco-Pereira, V., \& Gombosi, T. I. 2009a, ApJ, 703, 1734

Vidotto, A. A., Opher, M., Jatenco-Pereira, V., \& Gombosi, T. I. 2009b, ApJ, 699,441

Vidotto, A. A., Opher, M., Jatenco-Pereira, V., \& Gombosi, T. I. 2010, ApJ, 720,1262

Vourlidas, A., \& Howard, R. A. 2006, ApJ, 642, 1216

Walter, F. M., \& Kuhi, L. V. 1981, ApJ, 250, 254

Whelan, E. T., Dougados, C., Perrin, M. D., et al. 2010, ApJ, 720, L119

White, R. J., \& Basri, G. 2003, ApJ, 582, 1109

Wood, B. E. 2004, Living Rev. Sol. Phys., 1, 2

Wood, B. E., Müller, H.-R., Zank, G. P., Linsky, J. L., \& Redfield, S. 2005, ApJ, 628, L143

Yang, H., Herczeg, G. J., Linsky, J. L., et al. 2012, ApJ, 744, 121

Yashiro, S., Akiyama, S., Gopalswamy, N., \& Howard, R. A. 2006, ApJ, $650, \mathrm{~L} 143$

Yashiro, S., Gopalswamy, N., Akiyama, S., Michalek, G., \& Howard, R. A. 2005, J. Geophys. Res. (Space Phys.), 110, 12 